\title{
Effect of Solvents on the Morphological Characterization of Enteric Nanoparticles
}

\author{
Danay Rosa Dupeyrón Martell, Jacques Rieumont Briones ${ }^{\mathrm{b} *}$, \\ Mayra González, Hurtado , Alicia del Real López $z^{\mathrm{d}}$,Víctor Manuel Castaño Meneses ${ }^{\mathrm{d}}$ \\ anstituto de Ciencia y Tecnología de Materiales, Universidad de la Habana, Cuba \\ ${ }^{\mathrm{b}}$ Departamento de Química-Física, Facultad de Química, Universidad de la Habana, \\ Ciudad de la Habana, Cuba \\ ${ }^{\mathrm{c}}$ Centro de Ingeniería e Investigaciones Químicas, \\ Ciudad de la Habana, Cuba \\ ${ }^{\mathrm{d} C e n t r o ~ d e ~ F i ́ s i c a ~ A p l i c a d a ~ y ~ T e c n o l o g i ́ a ~ d e ~ A v a n z a d a, ~}$ \\ Universidad Nacional Autónoma de México \\ A.P. 1-1010 Santiago de Querétaro, Querétaro 7600 México
}

Received: March 18, 2009; Revised: September 24, 2009

\begin{abstract}
Size and external and internal morphologies of nanoparticles and microparticles are very important on the design of drug devices for controlled release. Random enteric copolymers such as poly (methacrylic acid-coethyl acrylate) and poly (methacrylic acid-co-methyl methacrylate) were used to produce nanoparticles, which contain a model drug and could be employed as drug carriers for proteins. The solvent effect on re-dispersion of such nanoparticles was studied by Scanning Electron Microscopy (SEM) and revealed not only differences in size, but also several shapes, depending on the chemical nature of the polymer matrix and the non-solvent used. Acrylate containing copolymers in acidic aqueous dispersions lead to spheroidal particles. However for the copolymer containing methyl methacrylate, spheroidal particles collapsed in a "grenade" type morphology and besides some cubic structures are also formed.

Dynamic Light Scattering (DLS) studies of the re-dispersed nanoparticles showed the strong tendency to form agglomerates not only in acidic water but also in hexane and the presence of bimodal size distributions.
\end{abstract}

Keywords: nanoparticles morphology, enteric copolymers, solvent effect, agglomerates

\section{Introduction}

Drug delivery is one of the most actives areas in today's nanotechnology and biotechnology. Indeed, from the pharmacological standpoint, an increasing number of proteinic drugs present some sort of drawbacks, in particular for oral formulations, due to their instability in the gastro intestinal tract ${ }^{1-4}$. In this regard, many attempts to face this challenge have been reported recently $y^{4,5}$ and one of them is by using an enteric copolymer ${ }^{6}$. The very concept of preparing small particles as carriers of core material trapped within a polymeric material dates back, at least, to the 1930s ${ }^{7}$. Ever since, a number of methods for preparing carrier microparticles have been developed, some based exclusively on physical or chemical phenomena, whereas others combine both physical and chemical phenomena. Recently, the use of nanoparticles has become increasingly important because their size and their surface properties offer many advantages, such as improved solubility, targetability and adhesion to tissue ${ }^{8-11}$. Controlling the size is also important because only certain particle size can be used for a specific use and release characteristics greatly depend on size and morphology of the particles involved.

There are numerous factors that can influence the morphology obtained with a specific drug/polymer combination, among them: the nature of the drug, the nature of the polymer, the amount of drug in the nanoparticles and the nature and amount of the emulsifier used in the aqueous phase. Amorphous polymers generally give particles with a smooth surface whereas semi-crystalline polymers did not ${ }^{12}$. The encapsulation process can also play its role in determining the external and internal morphology of particles. A process of double emulsion (water-oil-water) can induce multivesicular particles as has been reported from transmission electron microscopy studies ${ }^{13,14}$ and also the stability of the emulsion can induce morphological changes ${ }^{15,16}$.

The present work deals with the morphological characterization of nanoparticles obtained by using three different matrixes, two types of enteric copolymer and a blend of one of them with poly(ethylene glycol) (PEG). The enteric matrix was selected, according to a previous work ${ }^{6}$ using a model drug such as the bovine serum albumin (BSA) in order to protect protein from the lower $\mathrm{pH}$ of the stomach and enzymes, whereas the blend with PEG was used to enhance further the protection of the protein encapsulated ${ }^{6,17}$.

The main purpose of the present paper was to analyze by SEM the particle size and morphology changes induced by solvents on re-dispersion of nanoparticles and their diameter distribution by dynamic light scattering.

\section{Experimental}

\subsection{Materials and procedures}

- Bovine serum albumin (BSA) (MW 66 kD) was supplied by Sigma Chemical;

- Kollicoat ${ }^{\boxplus}$ MAE 100 P: Anionic copolymer based on methacrylic acid and ethyl acrylate (1:1), Tg: $110^{\circ} \mathrm{C}$ from BASF;

- Eudragit ${ }^{\circledast}$ L-100: Anionic copolymer of methacrylic acid and methyl methacrylate (1:1), MW: $135.000, \mathrm{Tg}>150{ }^{\circ} \mathrm{C}$, supplied by Röhm Pharma Polymers;

- Tween $80^{\circledast}$ used was supplied by Fluka Chemie AG (93781), 
viscosity $375-480 \mathrm{mPas}, \mathrm{n}^{20}{ }_{\mathrm{D}} 1,472$;

- Methanol, Hexane, and Xylene (reagent grade), supplied by MERCK;

- $0,2 \mu \mathrm{m}$ Filters (Sartorious AG PETF and Advantec MFS Nylon); and

- Ultra Turrax homogenizer (Model T- 25 IKA).

\subsection{Encapsulation procedure}

An aqueous solution ( $2 \mathrm{~mL}$ ), containing BSA (20 mg) and PEG (40 mg), was added to a copolymer solution in methanol $(20 \mathrm{~mL}$, $1 \%$ ) and the mixture was homogenized at $9500 \mathrm{rpm} / \mathrm{min}$, by using an Ultra Turrax homogenizer (Model T-25 IKA). Then, the dispersion obtained in this first step, was emulsified over a buffer solution $(120 \mathrm{~mL}, \mathrm{pH}=2)$ containing Tween 80 at $9500 \mathrm{rpm} / \mathrm{min}$. More details are described in a previous work ${ }^{6}$. Table 1 contains a description of all the samples prepared and the encapsulation parameters.

\subsection{Characterization techniques}

i) Scanning Electron Microscopy (SEM)

Samples obtained by the encapsulation procedure were ultrasonically re-dispersed for 10 minutes and dried and coated by sputtering with a layer of gold, of approximately $20 \mathrm{~nm}$. An EMS 550 Sputter coater was used. Samples were observed in a Scanning Electron Microscope (JEOL-JSM -6060LV). One hundred particles were random taken for each sample for evaluating the average diameter.

ii)Dynamic Light-Scattering (DLS).

Prior to use, all the solvents used were filtrated with $0.2 \mu \mathrm{m}$ filters to eliminate dust and the sample holder was cleaned with distilled water followed by acetone, to prevent contamination. For each sample, $2 \mathrm{mg}$ of particles were suspended in $20 \mathrm{~mL}$ of solvent and filtrated. Filters of $2 \mu \mathrm{m}$ Sartorious AG PTFE and Advantec MFS Nylon were used for organic and acid

Table 1. Experimental conditions for BSA encapsulation with the different matrixes.

\begin{tabular}{llcc}
\hline Sample & \multicolumn{1}{c}{ Polymer Matrix } & Surfactant amount $(\mathrm{mg})$ & Stirring rate $(\mathrm{rpm} / \mathrm{min})$ \\
\hline B6 & Kollicoat $^{\circledR}$ MAE 100 P & 20 & 9500 \\
B2- PEG & Kollicoat $^{\circledR}$ MAE 100 P + PEG & 20 & 9500 \\
B1 -L100 & Eudragit $^{\circledR}$ L100 & 20 & 9500 \\
\hline
\end{tabular}

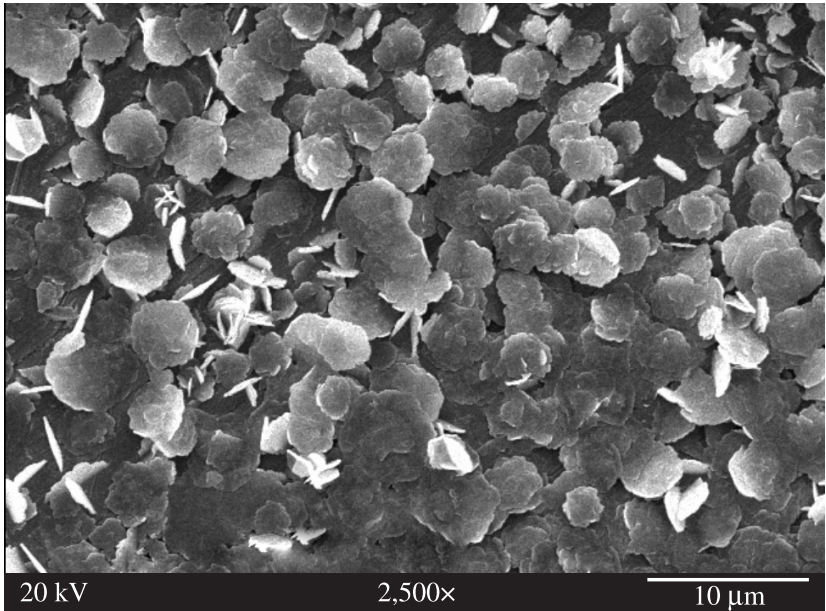

(a)

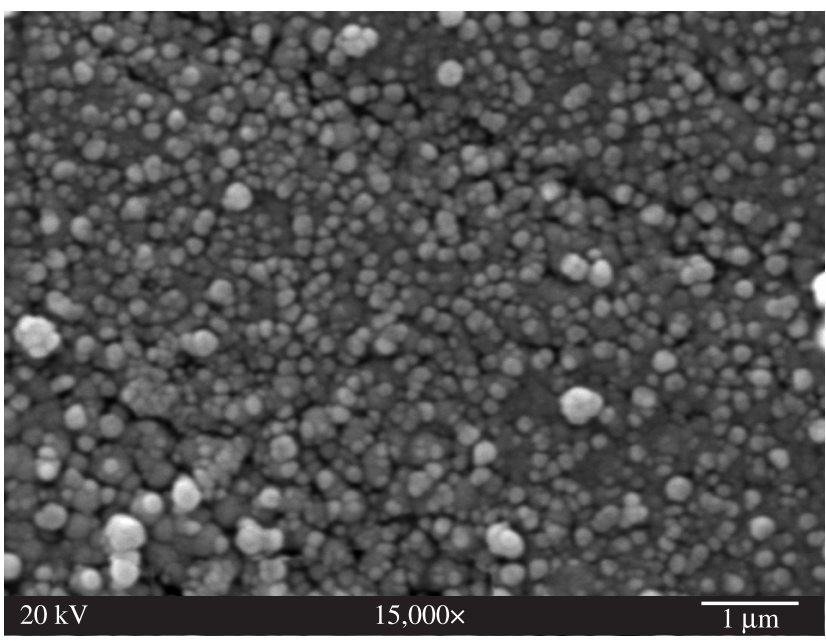

(c)

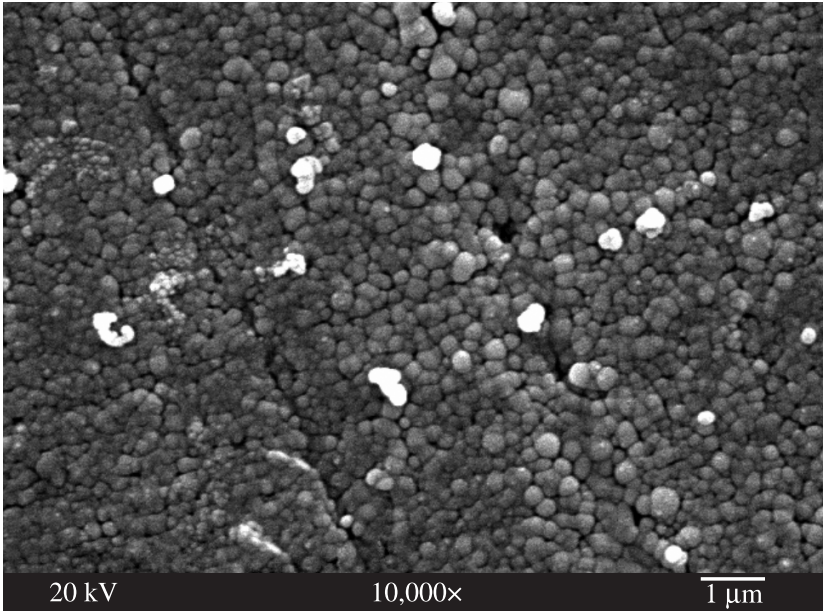

(b)

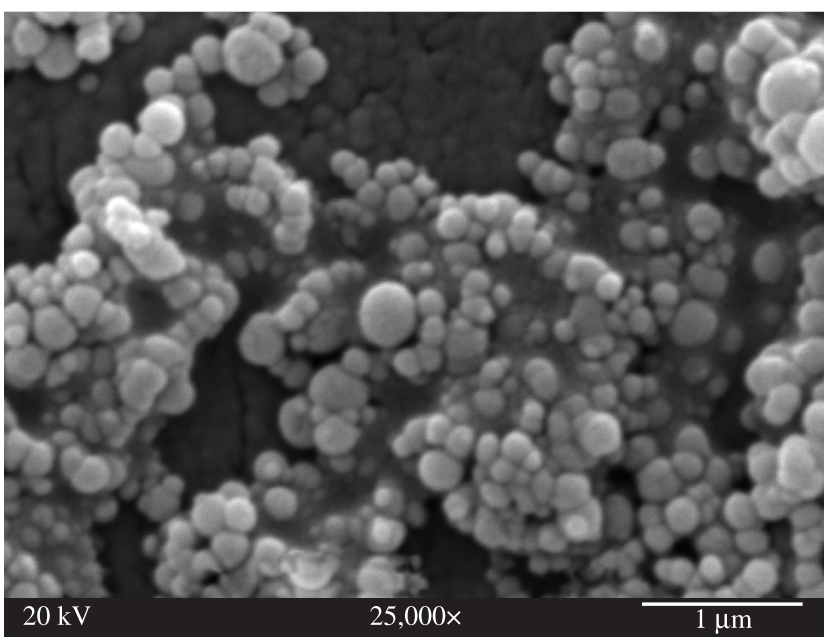

(d)

Figure 1. Scanning electron micrographs at different magnifications of sample B6: a) $2500 \times$ b) $10000 \times$ c) $15000 \times$ and d) $25000 \times$. 
aqueous solutions respectively. The samples were maintained in an ultrasonic bath for 10 minutes. The scattering cells (10 $\mathrm{mL}$ cylindrical vials) were immersed in a large-diameter thermostated bath of index-matching liquid (transdecalin). DLS measurements were performed in a Dynamic Light Scattering Brookhaven Instrument machine with a Model BI900 correlator. The results were analyzed by using the Non Negative Least Square (NNLS) and Contin methods ${ }^{18,19,20}$.

\section{Results and Discussion}

For comparison purposes all samples were obtained using identical procedures and amounts as is shown in Table 1. The control of particle size and shape depends mainly on parameters such as the amount of surfactant, the stirring rate and the chemical nature of the polymer matrix. Thus, surfactant and stirring rate were the same for all the experiments.

All the samples were obtained as described in the Experimental Part. By this procedure, the enteric copolymer closes their conformations to a more compact one and the protein is trapped by the polymer coating. Nanoparticles obtained were redispersed in some solvents such water at $\mathrm{pH}=2$ and hexane where the enteric copolymers are insoluble, and xylene that is a selective solvent for the hydrophobic units.

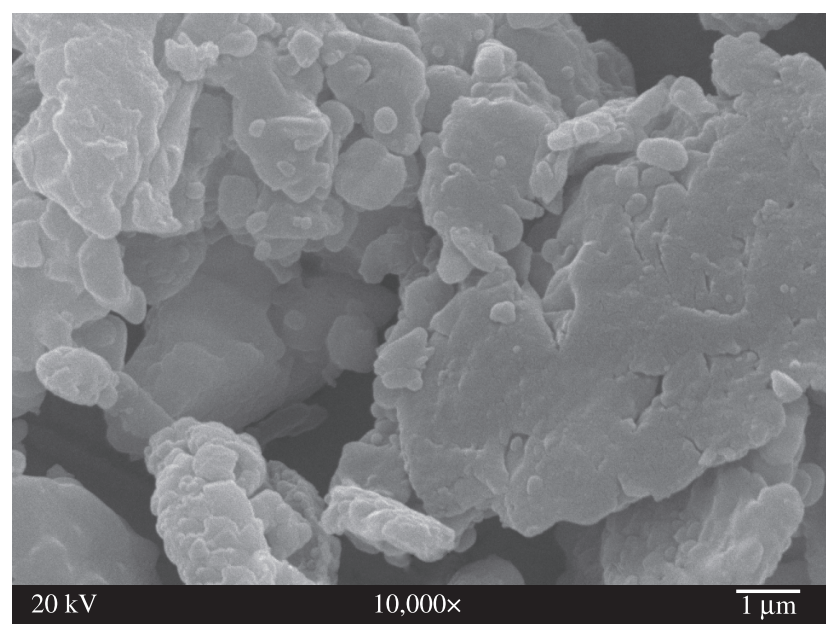

(a)

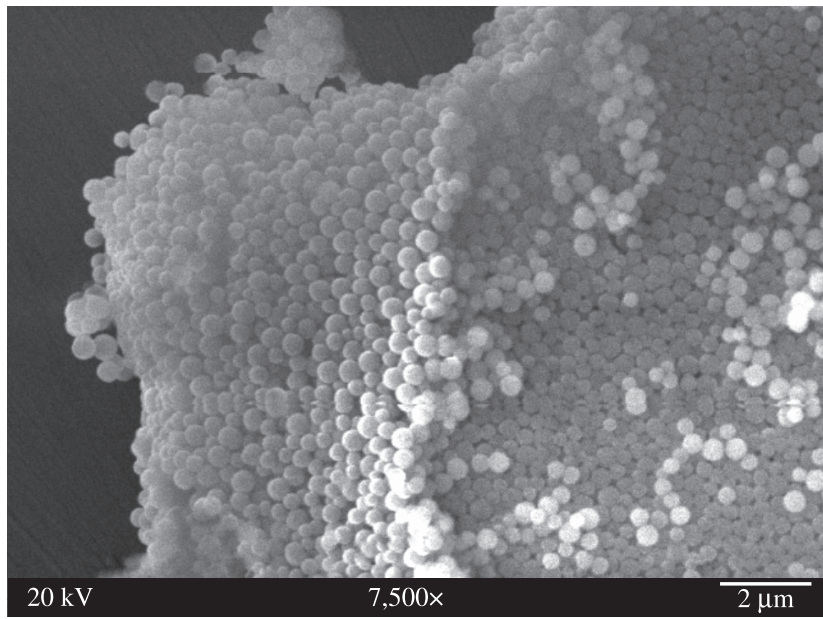

(c)
Nanoparticles behaviour with solvents will be discussed separately in the following items:

\subsection{Copolymer: poly (methacrylic acid-co-ethyl acrylate)}

In the case of sample B6 when re-dispersed in pure water, where particles can swell and even dissolve, a leaf-like morphology was observed (Figure 1a). However, when the same sample is re-suspended in an acidic aqueous medium where the copolymer is insoluble, the particles show spheroidal shapes (Figures 1b, 1c and 1d); surfaces are rough and agglomeration is present to some extent.

One feature of these enteric random copolymers is that contain polymethacrylic acid in their structure and the common drying processes do not exclude completely the water, due to its hydrophilic nature. Thus, it is unavoidable that traces of water remain inside the particle or near its surface and seem to act as plasticizers, contributing to agglomeration.

\subsection{Blend of the copolymer poly (methacrylic acid-co-ethyl acrylate) and poly (ethylene glycol)}

For sample B2-PEG re- dispersed in xylene, a better solvent for the acrylate units than for the methacrylic acid ones, a layered compact structure is shown (Figure 2a). However, re-suspension of this sample in hexane, a non-solvent for this copolymer, results in perfect spheres with smooth surfaces (Figures $2 \mathrm{c}$ and $2 \mathrm{~d}$ ). This interesting

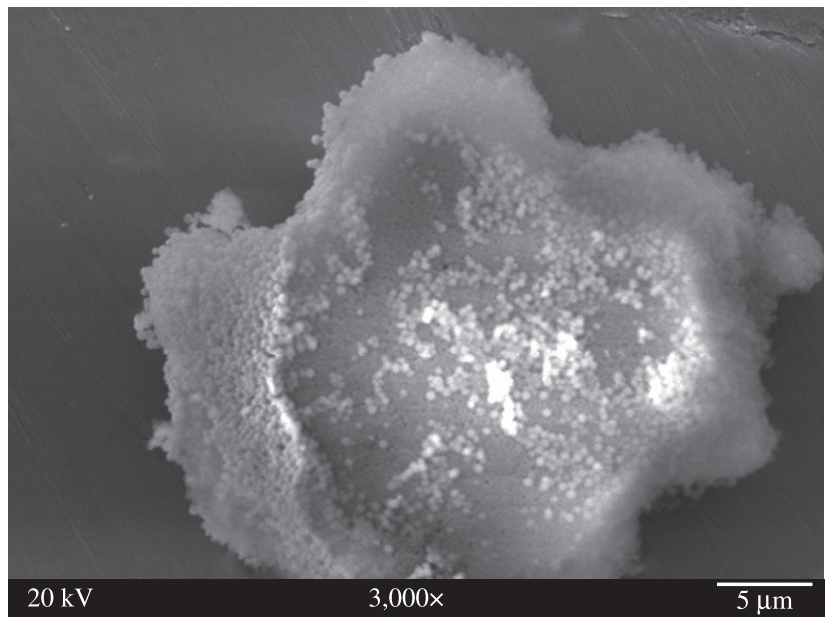

(b)

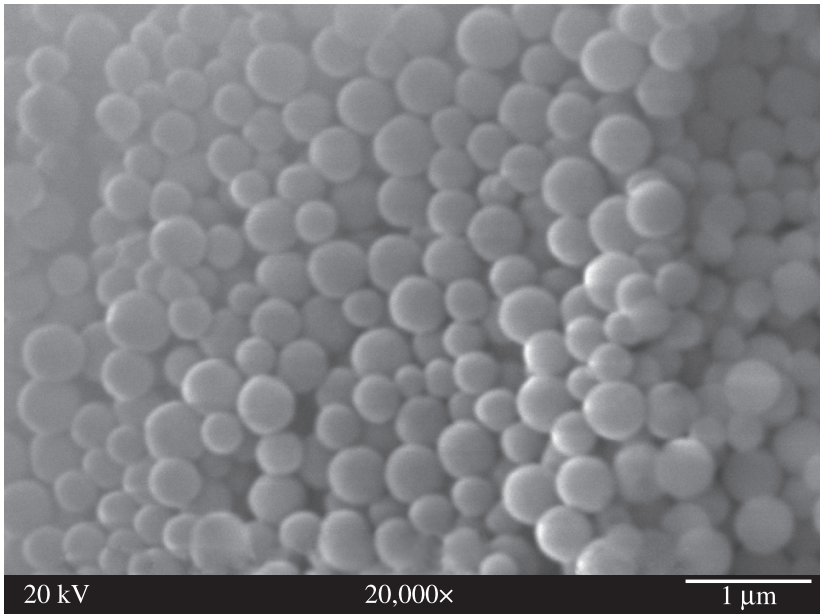

(d)

Figure 2. Scanning electron micrographs at different magnifications of sample B2-PEG: a) $10000 \times$ b) $3000 \times$ c) $7500 \times$ d) and $20000 \times$. 


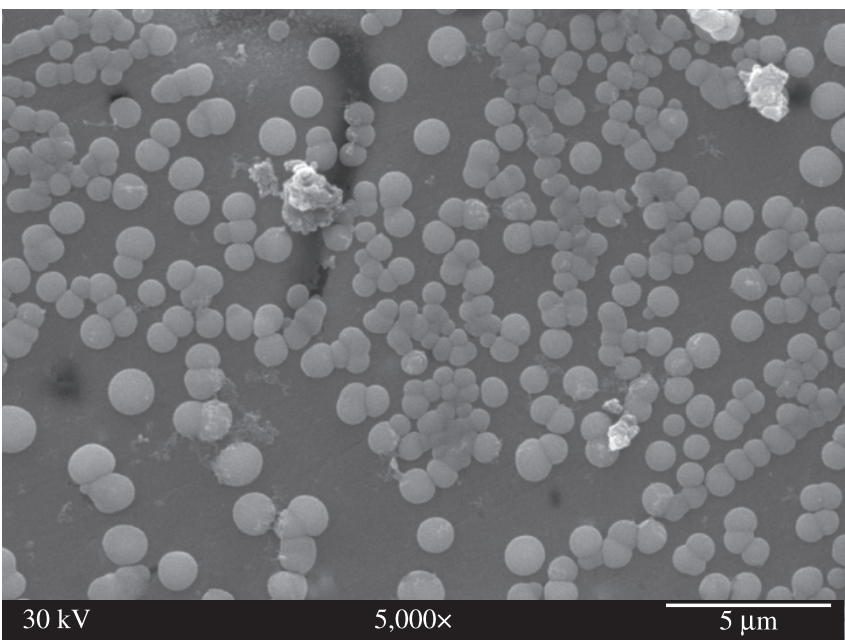

(a)

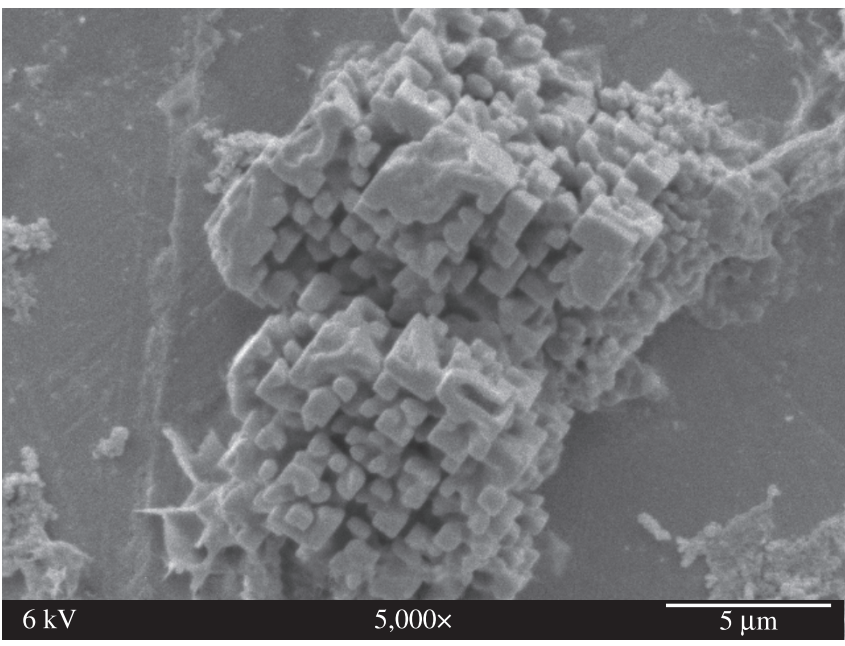

(c)

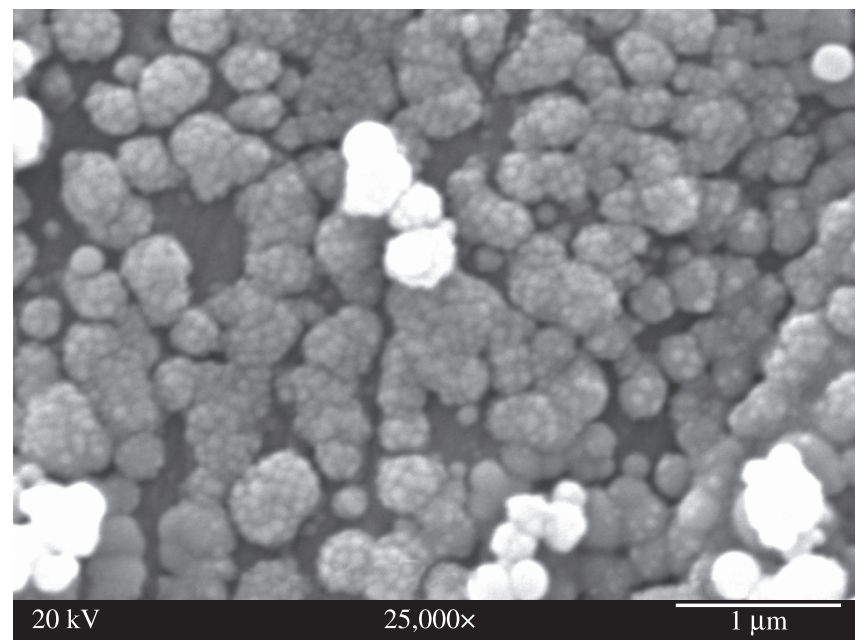

(b)

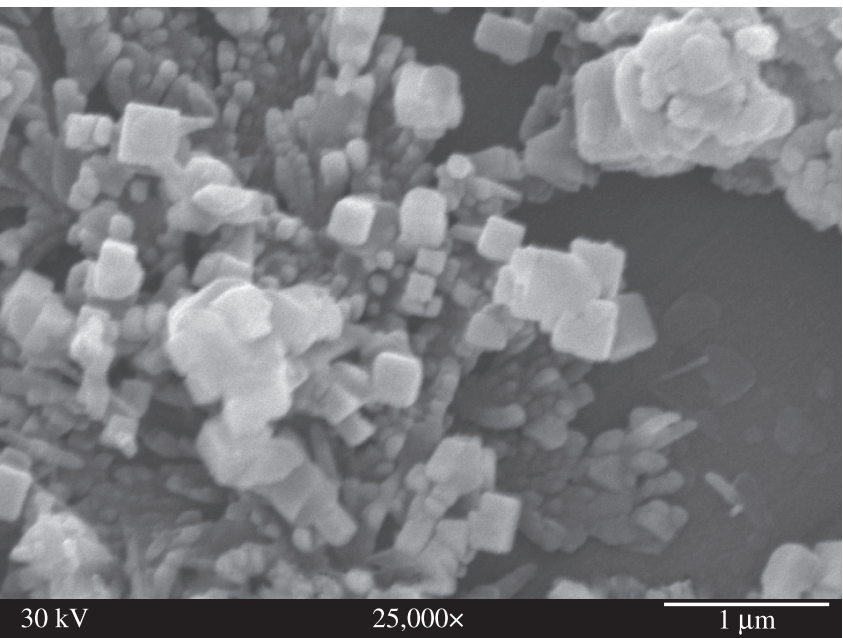

(d)

Figure 3. Scanning electron micrographs at different magnifications of sample B1-L100: a) spheroidal shape 5000× b) spheroidal shape $25000 \times$ c) cubic shape $5000 \times$ d) and cubic shape $25000 \times$.

result may be due to the extremely high insolubility of the copolymer in hexane, which leads to a more compact material. PEG is a semicrystalline polymer and the poly (methacrylic acid-co-ethyl acrylate) is amorphous. Amorphous polymers, as was said in the Introduction, give as a rule particles with smooth surfaces. Thus, possibly PEG is forming the core of particles.

Nevertheless, agglomeration is not ruled out completely as to be expected in hexane and some clusters, such as those shown in Figure $2 b$, are present. This material is a blend and differences on the rate of shrinkage of the two components could be producing such result.

\subsection{Copolymer: poly (methacrylic acid-co-methyl methacrylate)}

Sample B1 L100 shows interesting unique features. The suspension in acidic solution shows two types of morphologies (Figures 3a and $3 \mathrm{~d}$ ), namely spheroidal and cubic. These morphologies are produced simultaneously possibly due to the fact that the amount of surfactant for this copolymer or its hydrophilic-lypophilic balance, is not enough to stabilize all the spheres that can be formed. Furthermore, this copolymer possesses a higher glass temperature since it contains methyl methacrylate in its composition. Thus, this copolymer is stiffer in comparison to the other samples prepared with an ethyl acrylate-containing copolymer. It is well known that acrylates have lower glass temperatures than their methacrylates homologues and both are amorphous ${ }^{21,22}$. Those factors could make possible the formation of non-spheroidal structures such as the cubes observed, that can also agglomerate, forming separated groups (Figure 3c). Furthermore, a higher magnification micrograph of the spheroidal entities shows that they in fact are not "true" spheres, but the result of the compactation of smaller spheres, that produce a morphology resembling a "grenade" (see Figure 3b).

Those morphological changes are accompanied by changes in the particle diameters. Table 2 indicates that, under the same experimental nanoprecipitation conditions (amount of surfactant, stirring rates, acidic water); the composition of the enteric matrix practically determines not only the morphology but also the size of the particles. As a matter of fact the poly methacrylic acid-co-methyl methacrylate gave particles with greater diameters and more complex morphologies.

\subsection{Size distribution}

The tendency of these copolymers to agglomerate when redispersed was confirmed by light scattering. As can be seen in Table 3, particle diameters obtained by DLS are larger than the ones observed by SEM for all the samples as shown in Table 2. On the other hand, 
Table 2. Results obtained by SEM (D: average diameter; PDI: polydispersion index; SD: standard deviation; GSD: geometric standard deviation; CV: variance coefficient).

\begin{tabular}{lccccc}
\hline Sample & D $(\mathrm{nm})$ & PDI & \multicolumn{1}{c}{ SD } & GSD & CV\% \\
\hline B6 & 269.6 & 1.2 & 67.2 & 1.3 & 24.9 \\
B2-PEG & 423.9 & 1.1 & 64.2 & 1.2 & 15.2 \\
B1-L100 & 926.5 & 1.1 & 190.0 & 1.3 & 20.5 \\
\hline
\end{tabular}

Table 3. Results obtained by Dynamic Light Scattering.

\begin{tabular}{lcccc}
\hline Sample & $\begin{array}{c}\text { NNLS } \\
\text { Diameter }(\mu \mathrm{m})\end{array}$ & Deviation & $\begin{array}{c}\text { Contin } \\
\text { Diameter }(\mu \mathrm{m})\end{array}$ & Deviation \\
\hline B6 & 2.9 & 0.01 & 2.8 & 0.37 \\
B2-PEG & 6.9 & 0.04 & 5.2 & 0.44 \\
B1-L100 & 1.9 & 0.026 & 2.2 & 0.40 \\
\hline
\end{tabular}

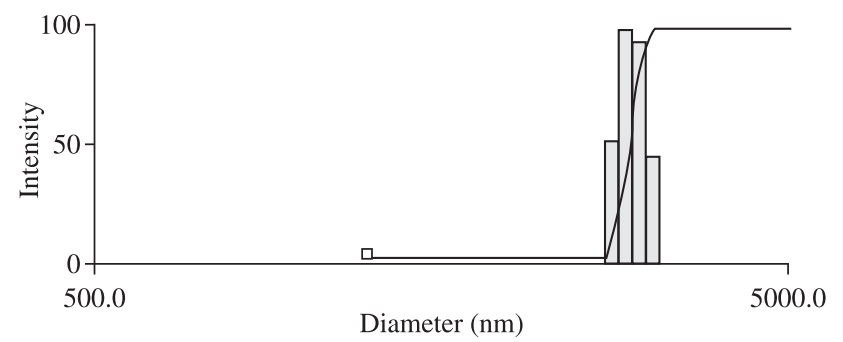

Figure 4. Results of Dynamic light Scattering of Sample B6. (NNLS)

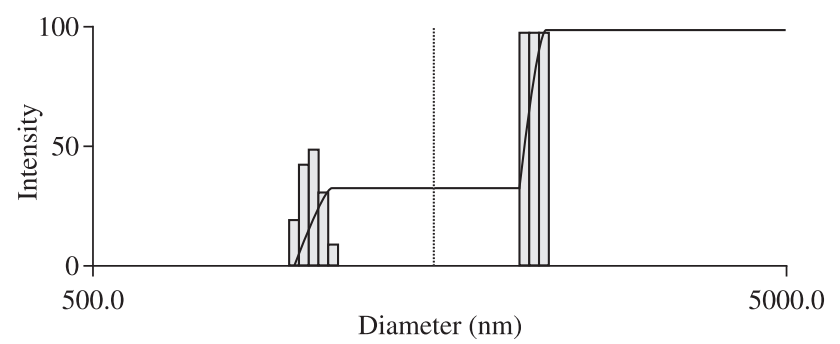

Figure 5. Results of Dynamic light Scattering of Sample B2 PEG. (NNLS)

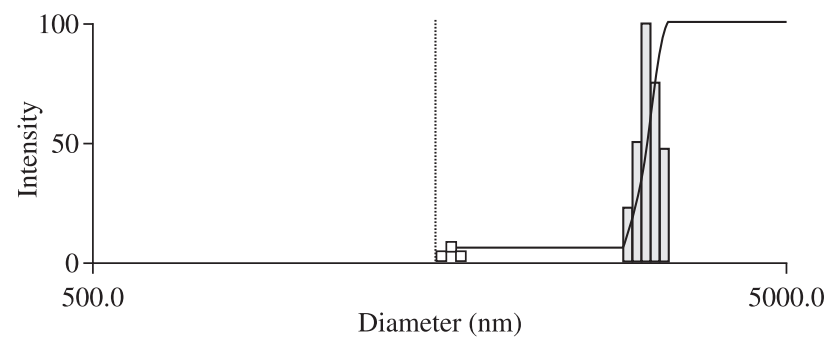

Figure 6. Results of Dynamic light Scattering of Sample B1L100. (NNLS)

Figure 4, 5 and 6 show the presence of two well-separated size groups, one corresponding to smaller particles and the other one to agglomerated clusters that are in much greater amounts than the first one. For the sample B2-PEG re-dispersed in hexane a bimodal distribution is even more noticeable as shown in Figure 5.

This is clearly in line with the Figure $3 \mathrm{~b}$ and $1 \mathrm{~d}$. It is an indication of a spontaneous process of self-aggregation due to the dual nature of these copolymers, which alters or biases the overall result ruled by the presence of hydrophilic and hydrophobic units in the copolymer. All those facts suggest that in the first step of the whole process nanoparticles are formed separately and in a second step become agglomerated.

\section{Conclusions}

SEM micrographs revealed how the specific polymeric matrix influences the morphology and size of nanoparticles obtained under the very same experimental conditions when are re-dispersed in differents solvents. In the particular case of the copolymer with methyl methacrylate that posseses the highest $\mathrm{Tg}$ value, two different shapes were observed, one spherical that resembles a grenade and the other one cubic. These morphologies are simultaneously produced under the same experiment. The dual character of these enteric materials (hydrophilic-hydrophobic) and their difference in physical properties seem to rule the complex behaviour observed.

It was also demonstrated by DLS the strong tendency of these copolymer nanoparticles to agglomerate and increase agglomerates sizes with re-dispersion, leading even to bimodal diameter distributions, more significantly when PEG is used in the matrix.

\section{Acknowledgements}

The authors are grateful to the Red de Macrouniversidades for financial support. We also thank Dra. Susana Vargas and Dr. Rogelio Rodríguez for their kind help with the DLS experiments and theory. The copolymers were a courtesy of Röhm Pharma Polymer.

\section{References}

1. Shen WC. Oral peptide and protein delivery: unfulfilled promises? Drug Discovery Today. 2003; 8(14):607-608.

2. Nakamura K, Murray R, Joseph J, Peppas N, Morishita M and Lowman A. Oral insulin delivery using $\mathrm{P}(\mathrm{MMA}-\mathrm{g}-\mathrm{EG})$ hydrogels effects on network morphology on insulin delivery characteristics. Journal of Controlled Release. 2004; 95(3):589-599.

3. Caliceti $\mathrm{P}$ and Veronese FM. Phamacokinetic and biodistribution properties on poly (ethylene glycol) protein conjugates. Advanced Drug Delivery Reviews. 2003; 55(10):1261-1277.

4. Whelan J. Beyond pegylation. Drug Discovery Today. 2005; 10(5):301-306.

5. Carino G, Jacob J and Mathiowitz E. Nanosphere based oral insulin delivery. Journal of Controlled Release. 2000; 65(1-2):261-269.

6. Dupeyrón D, González M, Sáez V, Ramón J and Rieumont J. Nanoencapsulation of protein using an enteric polymer as carrier. IEE Proceedings Nanobiotechnology. 2005; 152(5):165-168.

7. Benitas S. Microencapsulation: methods and industrial applications. New York: Marcel Dekker Inc.; 1996

8. Donini C, Robinson DN, Colombo P, Giordano F and Peppas NA. Preparation of poly (methacrylic acid-g-poly (ethylene glycol) nanospheres from methacrylic monomers for pharmaceutical applications. International Journal of Pharmaceutics. 2002; 245(1-2):83-91.

9. Kawashima Y. Preface nanoparticulate systems for improved drug delivery. Advanced Drug Delivery Reviews. 2001; 47(1):1-2.

10. Gonzalez M, Galano A, Rieumont J, Lopez T, Dupeyron D and Albaran L. Drug-Matrix interactions in nanostructured materials containing Acetyl Salicylic Acid Using an Enteric Polymer as a Coating. Journal of Physical Chemistry C. 2008; 112(51):20222-20226.

11. Gonzalez M, Rieumont J, Dupeyron D, Perdomo I, Abdón L and Castaño VM. Nanoencapsulation of acetyl salicylic acid within enteric polymer nanopartilcles. Review on Advanced Matererial Science. 2008; 17:71-75.

12. Martin M, Miguens FC, Rieumont J and Sanchez R. Tailoring of the external and internal morphology of poly-3-hydroxy butyrate microparticles. Colloid Surface. 2000; B17(2):111-116. 
13. Eligio T, Rieumont $\mathrm{J}$ and Sanchez R. Characterization of chemically modified poly (3-hydroxyalcanoates) and their performance as matrix for hormone release. Die Angewandte Makromolekulare Chemie. 1999; 270:69:70.

14. Schugens CH, Laruelle N, Nihant R, Grandphils C, Jérôme R and Teyssie P. Effect of the emulsion stability on the morphology and porosity of semicrystalline poly-L-lactide microparticles prepared by w/o/w double emulsion-evaporation. Journal of Controlled Release. 1994; 32(2):161176

15. Nihant N, Schugens CH, Grandphils C, Jérôme R and Teyssie P. Polylactide microparticles prepared by double emulsion/evaporation tecnique. 1. Effect of primary emulsion stability. Pharmaceutical Research. 1994; 11(10):1479-1484

16. Nihant N, Schugens CH, Grandphils C, Jérôme R and Teyssie P. Polylactide microparticles prepared by double emulsion-evaporation. 2 . Effect of the poly (lactide-co-glycolide) composition on the stability of the primary and secondary emulsions. Journal of Colloid and Interface Science. 1995; 173(1):55-65.
17. Ferrero C, Bravo I and Jiménez MR. Drug release kinetics and fronts movement studies from methyl methacrylate (MMA) copolymer matrix tablets: effect of copolymer type and matrix porosity. Journal of Controlled Release. 2003; 92(1-2):69-82.

18. Giacomelli C, Le-Men L, Borsali R, Lai-Hee-Him J, Brisson A, Armes $\mathrm{SP}$ and Lewis AL. Phosphorylcholine-based $\mathrm{pH}$-responsive diblock copolymer micelles as drug delivery vehicles: light scattering, electron microscopy, and fluorescence experiments. Biomacromolecules. 2006; 7(3):817-828

19. Provencher SW. Contin: a general purpose constrained regularization programm for inverting noisy linear algebraic and integral equations. Computer Physics Communications. 1982; 27:229-242.

20. Stock RS and Ray WH. Interpretation of photon-correlation spectroscopy data: a comparison of analysis methods. Journal of Polymer Science. Part B: Polymer Physics. 1985; 23(7):1393-1398.

21. Luskin L and Myers RJ. Acrylic Ester Polymers. In: Mark HF. (Ed.). Encyclopedia of Polymer Science and Technology. 1 ed. New York: John Wiley \& Sons; 1964. p. 246-328. (vol. 1).

22. Brandrup I and Immergut EH. Polymer Handbook. New York: Wiley InterScience; 1989. 